\title{
Consensus of multi-agent systems with nonuniform non-differentiable time-varying delays
}

\author{
H. J. Savino ${ }^{\star}$, A. P. L. Cota, F. O. Souza, L. C. A. Pimenta, E. M. A. M. Mendes ${ }^{\dagger}$ and L. A. Mozelli ${ }^{\ddagger}$
}

\begin{abstract}
In this paper the consensus problem for continuous time multi-agent systems in the presence of time-delay is addressed. A novel sufficient condition for the case of nonuniform non-differentiable time-varying delays with minimum value greater than zero and a method to compute an estimate of the convergence rate are given. Simulation examples are given to show the performance of the proposed method.
\end{abstract}

\section{INTRODUCTION}

The study of distributed multi-agent systems has called the attention of several research groups in the past few years. This is mainly due to the vast number of applications that may benefit from the results in this field. For an interesting review of recent results refer to [1].

An important problem in distributed multi-agent systems is the one of reaching a group agreement. More specifically, let $x_{i}$ be the value of a physical variable associated to agent $v_{i}$, where $i=1,2, \ldots, n$. This physical variable may be the agent height, position, velocity, orientation, etc. depending on the application. We say that agent $v_{i}$ is in agreement with agent $v_{j}$ if and only if $x_{i}=x_{j}$. Moreover, if all agent values reach a common value, we say that there is a group agreement (consensus).

One of the main concerns of researchers in this field is the derivation of techniques that adequately cope with the number of agents. Usually in this case only local information should be taken into account by agent control laws (consensus protocol) to solve the consensus problem. This means that each agent should compute its actions based on information provided by only a subset of the group. Several works have studied these local information based control laws to solve consensus problem in different scenarios such as double integrator dynamics [2], input saturation [3], etc.

In the present paper we are interested in the case where information exchange between agents cannot happen instantaneously due to the characteristics of the available communication channels. This is important since most real applications rely on a computer network to provide this

* Programa de Pós-Graduação em Engenharia Elétrica - Universidade Federal de Minas Gerais - Av. Antônio Carlos 6627, 31270-901, Belo Horizonte, MG, Brasil. heitorjs@ufmg.br. This work has been supported by the Brazilian agency CAPES. Partially supported by CNPq-Brazil.

$\dagger$ Departamento de Eng. Eletrônica, Universidade Federal de Minas Gerais, Av. Antônio Carlos 6627, 31270-010, Belo Horizonte, MG, Brasil. aplc@ufmg.br, fosouza@cpdee.ufmg.br, lucpim@cpdee.ufmg.br, emmendes@cpdee.ufmg.br. Partially supported by the FAPEMIG, CNPq and CAPES.

¥ Departamento de Eng. de Telecomunicações e Mecatrônica, Universidade Federal de São João del-Rei, Rod. MG 443, km 7, 36420-000 - Ouro Branco, MG, Brasil. mozelli@ufs j.edu.br. Partially supported by the FAPEMIG, CNPq and CAPES. information exchange, and such a network may be subject to delays. More specifically, we are interested in the case of fixed communication topology, continuous-time multi agent systems in which both the state information of neighbor robots and the state information of the agent itself are subject to delay.

In the case of constant uniform time-delay, a necessary and sufficient condition for average consensus was presented in [4]. Considering the same time-delay configuration, [5] obtained a sufficient condition in the presence of external disturbance and model uncertainty. Systems subject to uniform differentiable time-varying delays were studied in [6]. Other works, such as [7] and [8], studied nonuniform differentiable time-varying delays. By nonuniform, we mean there may be a different time-delay value for each communication channel. In [9] and [10] the authors also considered the possibility of nonuniform non-differentiable time-varying delays that satisfy $0 \leq \tau_{i j}(t) \leq h_{i j}$, where $h_{i j}>0$ are constants.

In this work, differently from previous works found in the literature, we present a novel sufficient condition for consensus in the context of single integrator agents and nonuniform non-differentiable time-varying time-delays that satisfy $\tau_{i j}(t) \in\left[\tau-\mu_{m}, \tau+\mu_{m}\right]$, where $\tau>0$ is a constant, $\mu_{m}$ is also a constant, and $\tau_{i j} \geq 0$. Note that in this case $\tau_{i j}(t)$ may have a minimum value greater than zero, which can be applied in cases where the time-delay has an estimated value, varying in a given range. This is important since it will allow to show consensus in different regions in the domain of time-delay [11]. Other methods would find only the region in the domain of time-delay where the minimum value of $\tau_{i j}(t)$ is zero. In addition to that, a method to compute an estimate of the convergence rate is also presented. Finally, our linear matrix inequality (LMI) condition provides similar results to those obtained when considering other recently published conditions in the simple case of single constant time-delay.

Next section reviews some necessary background theory. In Section III we show how the consensus problem can be transformed to a stability problem. By using techniques that provide conditions for the stability of systems with timedelay we devise a novel sufficient condition for consensus in Section IV. Simulations showing the efficacy of the of proposed methodology are presented in Section V. Finally, conclusions are given in Section VI.

\section{BACKGROUND}

\section{A. Algebraic Graph Theory}

Let $\mathcal{G}(\mathcal{V}, \mathcal{E}, \mathcal{A})$ be a simple undirected graph of order $n$, where $\mathcal{V}=\left\{v_{1}, \ldots, v_{n}\right\}$ is the set of vertices, $\mathcal{E}$ is the set 
of edges which have no orientation, and $\mathcal{A}=\left[a_{i j}\right]$ is the weighted adjacency matrix. Let $\mathcal{I}=\{1,2, \ldots, n\}$ be the set of graph node indexes. The elements of the adjacency matrix are given by: (i) $a_{i j}=0$ if $i=j$ or if there is no edge connecting $v_{i}$ to $v_{j}$; and (ii) $a_{i j}=a_{j i}>0$ if and only if there exists a link between $v_{i}$ and $v_{j}$.

The degree matrix, $\Delta=\left[\Delta_{i j}\right]$, is a diagonal matrix with $\Delta_{i j}=\sum_{j=1}^{n} a_{i j}$. The Laplacian matrix associated with the graph is then defined as $L=\Delta-\mathcal{A}$.

A graph is called connected if there is a path from each node to every other node. A path is a sequence of graph edges of the form $\left(v_{i 1}, v_{i 2}\right),\left(v_{i 2}, v_{i 3}\right), \ldots$, where $v_{i k} \in \mathcal{V}$.

Next lemma regarding connected graphs will be useful in later sections:

Lemma 1: [12] If a graph $\mathcal{G}$ is connected then its Laplacian satisfies the following conditions:

1) There exists a unique eigenvalue of $L$ equal to zero, and $\mathbf{1}_{n}$ is the corresponding eigenvector, i.e., $L \mathbf{1}_{n}=0$.

2) The remaining $(n-1)$ eigenvalues are all real and positive.

\section{B. Consensus Protocol}

In this work, a multi-agent system with information exchange model given by a simple undirected graph is considered. An agent $i$ is represented by a graph node (vertex) $v_{i}$ and the communication channels between agents are represented by graph edges. Nodes sharing an edge are said to be neighbors. Each agent is assumed to have the following simple dynamics

$$
\dot{x}_{i}(t)=u_{i}(t), \quad i=1,2, \ldots, n
$$

where $x_{i} \in \mathbb{R}$ is the state variable of agent $i$ and $u_{i} \in \mathbb{R}$ is the control input.

It is well known that the following protocol [4] solves the consensus problem if information can be exchanged instantaneously:

$$
u_{i}=-\sum_{v_{j} \in N_{i}} a_{i j}\left(x_{i}-x_{j}\right)
$$

where $N_{i}$ is the set of neighbors of node $v_{i}$. It should be mentioned that $v_{i}$ and $v_{j}$ are neighbors if and only if $a_{i j} \neq 0$.

In this work, we consider the presence of time-delay during information exchange and the following protocol is studied:

$$
u_{i}=-\sum_{v_{j} \in N_{i}} a_{i j}\left(x_{i}\left(t-\tau_{i j}(t)\right)-x_{j}\left(t-\tau_{i j}(t)\right)\right)
$$

where $\tau_{i j}(t)$ is the time-delay in the communication between $v_{i}$ and $v_{j}$. It should be clear that we assume different delays for different communication channels. Moreover, we consider that this delay is independent of the information direction, i.e., $\tau_{i j}(t)=\tau_{j i}(t)$. It should be also emphasized that, in this work, the considered time-delays may be timevarying and non-differentiable.
Similarly to [10] and considering (1) and (3) the system dynamics may be written as:

$$
\dot{x}(t)=-\sum_{k=1}^{r} L_{k} x\left(t-\tau_{k}(t)\right)
$$

where $r$ is the number of different delays satisfying $r \leq$ $n(n-1) / 2$, and $L_{k}$ is the Laplacian matrix associated with the graph connections with delay $\tau_{k}(t)$.

\section{Stability Problem Formulation}

The protocol $u_{i}$ given in (3) will solve the consensus problem if and only if: $\lim _{t \rightarrow \infty}\left|x_{i}-x_{j}\right|=0, \quad \forall(i, j)$. In this section, we show how the consensus problem can be translated into a stability problem. Basically, this is done by using the same strategy presented in [5].

Let $\Psi_{n}$ be the matrix:

$$
\Psi_{n}=\left[\begin{array}{cccc}
n-1 & -1 & \ldots & -1 \\
-1 & n-1 & \ldots & -1 \\
\vdots & \vdots & \ddots & \vdots \\
-1 & -1 & \ldots & n-1
\end{array}\right],
$$

where $\Psi_{n}$ has $n-1$ eigenvalues equal to $n$ and a zero eigenvalue [5]. Let $U$ be an orthogonal matrix representing the eigenvectors of $\Psi_{n}$. This matrix can be written as $U=\left[\begin{array}{ll}U_{1} & \bar{U}_{1}\end{array}\right]$, where each column of $U_{1}$ is an eigenvector associated with the eigenvalue at $n$ and $\bar{U}_{1}$ is an eigenvector associated with the zero eigenvalue of $\Psi_{n}$. Therefore

$$
U_{1}^{T} \bar{U}_{1}=0
$$

and the following properties can be verified

$$
\begin{aligned}
\Psi_{n} U_{1} & =n U_{1} \\
\Psi_{n} \bar{U}_{1} & =0 \\
\bar{U}_{1} & =\alpha\left[\begin{array}{ll}
1 & 1 \ldots 1
\end{array}\right]^{T} \\
\bar{U}_{1} & =\alpha \mathbf{1}_{n},
\end{aligned}
$$

where $\alpha$ is a constant. Moreover,

$$
\begin{aligned}
\bar{U}_{1}^{T} \bar{U}_{1} & =1 \\
\alpha^{2} \mathbf{1}_{n}^{T} \mathbf{1}_{n} & =1 \\
\alpha^{2} n & =1 \\
\alpha & =\frac{1}{\sqrt{n}},
\end{aligned}
$$

thus,

$$
\bar{U}_{1}=\frac{\mathbf{1}_{n}}{\sqrt{n}} .
$$

Now, consider the following transformation:

$$
\left[\begin{array}{c}
z(t) \\
\bar{z}(t)
\end{array}\right]=\left[\begin{array}{c}
U_{1}^{T} \\
\bar{U}_{1}^{T}
\end{array}\right] x(t)=U^{T} x(t) .
$$

After computing the derivative we obtain:

$$
\left[\begin{array}{c}
\dot{z}(t) \\
\dot{\bar{z}}(t)
\end{array}\right]=U^{T} \dot{x}(t) \text {. }
$$

Due to Lemma 1, the Laplacian matrix $L_{k}$ in (4) satisfies: $L_{k} \mathbf{1}_{n}=0$ and $\mathbf{1}_{n}^{T} L_{k}=0$ (since $a_{i j}=a_{j i}$ ). By also 
considering the dynamics in (4) and equation (10), the system in (11) becomes:

$$
\begin{aligned}
{\left[\begin{array}{c}
\dot{z}(t) \\
\dot{\bar{z}}(t)
\end{array}\right] } & =-U^{T} \sum_{k=1}^{r} L_{k} x\left(t-\tau_{k}(t)\right) \\
& =-\sum_{k=1}^{r} U^{T} L_{k} U\left[\begin{array}{l}
z\left(t-\tau_{k}(t)\right) \\
\bar{z}\left(t-\tau_{k}(t)\right)
\end{array}\right] \\
& =-\sum_{k=1}^{r}\left[\begin{array}{cc}
\bar{L}_{k} & 0 \\
0 & 0
\end{array}\right]\left[\begin{array}{l}
z\left(t-\tau_{k}(t)\right) \\
\bar{z}\left(t-\tau_{k}(t)\right)
\end{array}\right]
\end{aligned}
$$

where $\bar{L}_{k}=\left[\bar{l}_{k i j}\right]$ and $\bar{L}_{k}=U_{1}^{T} L_{k} U_{1}$.

In the next lemma we show that the group consensus in the case of the system in (4) can be achieved by studying the stability of part of the system in (12):

$$
\dot{z}(t)=-\sum_{k=1}^{r} \bar{L}_{k} z\left(t-\tau_{k}(t)\right)
$$

where $\bar{L}_{k} \in \mathbb{R}^{(n-1) \times(n-1)}$.

Lemma 2: If the system in (13) reaches the origin, then the system defined in (4) reaches consensus.

Proof: Consider the system output given by $y(t)=$ $C x(t)$, where $C=\frac{1}{n} \Psi_{n}$. Note that this output represents the distance of the agent state variables from the average value: $y=C x=\left[x_{1}^{T}-\bar{x}^{T}, x_{2}^{T}-\bar{x}^{T}, \cdots\right]^{T}$, where $\bar{x}=\frac{1}{n} \sum_{i=1}^{n} x_{i}$. Thus, consensus is achieved if and only if $y=\mathbf{0}$. By using the identities in (7):

$$
\begin{aligned}
y(t) & =C U U^{T} x(t) \\
& =C U\left[\begin{array}{l}
z(t) \\
\bar{z}(t)
\end{array}\right] \\
& =\frac{1}{n} \Psi_{n} U\left[\begin{array}{l}
z(t) \\
\bar{z}(t)
\end{array}\right] \\
& =\frac{1}{n}\left[\begin{array}{ll}
n U_{1} & 0
\end{array}\right]\left[\begin{array}{l}
z(t) \\
\bar{z}(t)
\end{array}\right]=U_{1} z(t)
\end{aligned}
$$

Since matrix $U_{1}$ is composed of linearly independent vectors the nullity of its null space $\mathcal{N}\left(U_{1}\right)$ is zero. Therefore $y(t)=\mathbf{0}$ if and only if $z(t)=\mathbf{0}$.

Next section presents an LMI condition for stability analysis of the system in (13).

\section{Consensus Analysis}

Definition 1: The multi-agent system in (4) achieves consensus with exponential convergence rate $\delta$ if

$$
\|z(t)\| \leq \sqrt{\frac{\rho(\delta)}{\lambda_{\min }\{P\}}} e^{-\delta t}, \quad \forall t \geq 0,
$$

where $z(t)$ is given in (10), $\lambda_{\min }\{P\}$ is the minimum eigenvalue of a symmetric real positive matrix $P$ and $\delta$ and $\rho(\delta)$ are positive scalars.

Theorem 1: Let be given $\tau>0, \mu_{m}$ and $\delta>0$. Then the system in (4) with all $\tau_{k}(t) \in\left[\tau-\mu_{m}, \tau+\mu_{m}\right]$ achieves consensus with exponential consensus rate $\delta$, if there exist real matrices: $F, G, P=P^{T}, S=S^{T}, Q, R_{1}=R_{1}^{T}, R_{2}$,
$R_{3}=R_{3}^{T}$ and $Z_{k}=Z_{k}^{T}, k=1,2, \ldots, r$, of dimension $(n-1) \times(n-1)$, such that the following LMIs are satisfied

$$
\left[\begin{array}{rr}
P & Q \\
* & \frac{e_{1}}{\tau} S
\end{array}\right]>0
$$

where $e_{1}=e^{-2 \delta \tau}$,

$$
\bar{R}=\left[\begin{array}{cc}
R_{1} & R_{2}^{T} \\
R_{2} & R_{3}
\end{array}\right]>0
$$

and

$$
\left[\begin{array}{c|c}
\Theta & \operatorname{row}\left\{\Gamma_{k}\right\} \\
\hline * & -e^{2 \delta\left(\mu_{m}+\tau\right)} \mu_{m} \operatorname{diag}\left\{Z_{k}\right\}
\end{array}\right]<0
$$

where $\Theta$ is given in (18), on the top of the next page, $\operatorname{row}\left\{\Gamma_{k}\right\}$ is a block-row matrix, i.e., $\operatorname{row}\left\{\Gamma_{k}\right\}=$ $\left[\Gamma_{1}, \ldots, \Gamma_{r}\right]$, with

$$
\Gamma_{k}^{T}=\mu_{m} \bar{L}_{k}^{T}\left[\begin{array}{llll}
F^{T} & G^{T} & 0 & 0
\end{array}\right]
$$

and $\operatorname{diag}\left\{Z_{k}\right\}$ is a block diagonal matrix with diagonal blocks $Z_{1}, Z_{2}, \ldots, Z_{r}$.

Proof: Consider the following Lyapunov-Krasovskii functional candidate:

$$
\begin{aligned}
V\left(z_{t}\right)= & e^{2 \delta t} z^{T}(t) P z(t)+2 e^{2 \delta t} z^{T}(t) \int_{t-\tau}^{t} Q z(\xi) d \xi \\
& +\int_{-\tau}^{0} \int_{t+s}^{t} e^{2 \delta \xi} \bar{z}^{T}(\xi) \bar{R} \bar{z}(\xi) d \xi d s \\
& +\int_{-\tau}^{0} e^{2 \delta(t+\xi)} z^{T}(t+\xi) S z(t+\xi) d \xi \\
& +\sum_{k=1}^{r} \int_{-\mu_{m}}^{\mu_{m}} \int_{t+s-\tau}^{t} e^{2 \delta \xi} \dot{z}^{T}(\xi) Z_{k} \dot{z}(\xi) d \xi d s
\end{aligned}
$$

where $\bar{z}^{T}(\xi)=\left[z^{T}(\xi) \dot{z}^{T}(\xi)\right], P=P^{T}, S=S^{T}, Q, R_{1}=$ $R_{1}^{T}, R_{2}, R_{3}=R_{3}^{T}$ and $Z_{k}=Z_{k}^{T}, k=1,2, \ldots, r$, and $z_{t}$ corresponds to the value of $z(\theta)$ with $\theta \in\left[t-\tau-\mu_{m}, t\right]$.

Initially, we show that the condition $V\left(z_{t}\right) \geq \epsilon|| z(t) \|(\epsilon>$ $0)$ is satisfied.

Assume that $S>0$ (implicit in (15)) and using Jensen Inequality [13, Prop. B.8] yields

$$
\begin{aligned}
& \int_{-\tau}^{0} e^{2 \delta(t+\xi)} z^{T}(t+\xi) S z(t+\xi) d \xi \\
& \quad \geq e^{2 \delta(t-\tau)} \int_{-\tau}^{0} z^{T}(t+\xi) S z(t+\xi) d \xi \\
& \quad \geq \frac{e^{2 \delta(t-\tau)}}{\tau} \int_{-\tau}^{0} z^{T}(t+\xi) d \xi \cdot S \cdot \int_{-\tau}^{0} z(t+\xi) d \xi
\end{aligned}
$$

Therefore, based on the previous inequality, we have that

$$
\begin{aligned}
V\left(z_{t}\right) \geq & \eta^{T}\left[\begin{array}{cc}
P & Q \\
* & \frac{e_{1}}{\tau} S
\end{array}\right] \eta+\int_{-\tau}^{0} \int_{t+s}^{t} e^{2 \delta \xi} \bar{z}^{T}(\xi) \bar{R} \bar{z}(\xi) d \xi d s \\
& +\sum_{k=1}^{r} \int_{-\mu_{m}}^{\mu_{m}} \int_{t+s-\tau}^{t} e^{2 \delta \xi} \dot{z}^{T}(\xi) Z_{k} \dot{z}(\xi) d \xi d s
\end{aligned}
$$

with $\eta^{T}=\left[\begin{array}{ll}e^{\delta t} z^{T}(t) & e^{\delta t} \int_{-\tau}^{0} z^{T}(t+\xi) d \xi\end{array}\right]$.

Therefore, if the right side of the previous equation is positive, it yields a sufficient condition to guarantee that 


$$
\Theta=\left[\begin{array}{cccc}
2 \delta P+Q+Q^{T}+\tau R_{1}-\frac{e_{1}}{\tau} R_{3}+S & * & * & * \\
-e_{2} F^{T}+P+\tau R_{2} & -e_{2}\left(G+G^{T}\right)+\tau R_{3}+2 \mu_{m} \mathcal{Z} & * & * \\
-e_{2} \overline{\mathcal{L}}^{T} F^{T}-Q^{T}+\frac{e_{1}}{\tau} R_{3} & -e_{2} \overline{\mathcal{L}}^{T} G^{T} & -e_{1}\left(\frac{1}{\tau} R_{3}+S\right) & * \\
2 \delta Q^{T}-\frac{e_{1}}{\tau} R_{2}^{T} & Q^{T} & \frac{e_{1}}{\tau} R_{2}^{T} & -\frac{e_{1}}{\tau} R_{1}
\end{array}\right]
$$

with $e_{1}=e^{-2 \delta \tau}, e_{2}=e^{-2 \delta\left(\mu_{m}+\tau\right)}, \overline{\mathcal{L}}=\sum_{k=1}^{r} \bar{L}_{k}$ and $\mathcal{Z}=\sum_{k=1}^{r} Z_{k}$.

$V\left(z_{t}\right) \geq \epsilon\|z(t)\|(\epsilon>0)$. Moreover, the LMI in (16) guarantees that $\bar{R}>0$ and the LMI in (17) implies that $Z_{k}>0$, for all $k$. Thus, if the LMIs in (15), (16) and (17) are satisfied, then $V\left(z_{t}\right) \geq \epsilon\|z(t)\|(\epsilon>0)$.

Now it is shown that the functional in (20) satisfies the derivative condition: $\dot{V}\left(z_{t}\right) \leq-\epsilon\|z(t)\|(\epsilon>0)$ if the LMI in (17) and $\bar{R}>0$ hold. For this purpose it is introduced a null term, considering the system in (13) and two matrices with appropriate dimensions, $F$ and $G$ :

$$
\begin{aligned}
0= & {\left[z^{T}(t) F+\dot{z}^{T}(t) G\right]\left[-\dot{z}(t)-\sum_{k=1}^{r} \overline{L_{k}} z\left(t-\tau_{k}(t)\right)\right] } \\
= & 2 e^{2 \delta\left(t-\tau-\mu_{m}\right)}\left[z^{T}(t) F+\dot{z}^{T}(t) G\right] \\
& \times\left[-\dot{z}(t)-\sum_{k=1}^{r} \bar{L}_{k}\left(z(t-\tau)-\int_{-\tau_{k}(t)}^{-\tau} \dot{z}(t+\xi) d \xi\right)\right] \\
= & 2 e^{2 \delta\left(t-\tau-\mu_{m}\right)}\left[z^{T}(t) F+\dot{z}^{T}(t) G\right] \\
& \times\left[-\dot{z}(t)-\sum_{k=1}^{r} \bar{L}_{k} z(t-\tau)\right]+v(t)
\end{aligned}
$$

where $v(t)=2 \Lambda \sum_{k=1}^{r} \overline{L_{k}} \int_{-\tau_{k}(t)}^{-\tau} e^{\delta\left(t-\tau-\mu_{m}\right)} \dot{z}(t+\xi) d \xi$ with $\Lambda=e^{\delta\left(t-\tau-\mu_{m}\right)}\left[z^{T}(t) F+\dot{z}^{T}(t) G\right]$.

Then, using the well known inequality

$$
2 a^{T} b \leq a^{T} X a+b^{T} X^{-1} b
$$

where $a, b \in \mathbb{R}^{n}$ and $X>0 \in \mathbb{R}^{n \times n}$. The following upper bound for $v(t)$ in (21) is obtained

$$
\begin{aligned}
v(t) \leq & \sum_{k=1}^{r} \int_{-\tau_{k}(t)}^{-\tau}\left(\Lambda \overline{L_{k}}\right) Z^{-1}\left(\Lambda \overline{L_{k}}\right)^{T} d \xi \\
& +\sum_{k=1}^{r} \int_{-\tau_{k}(t)}^{-\tau} e^{2 \delta\left(t-\tau-\mu_{m}\right)} \dot{z}^{T}(t+\xi) Z_{k} \dot{z}(t+\xi) d \xi \\
\leq & \sum_{k=1}^{r} \mu_{m}\left(\Lambda \overline{L_{k}}\right) Z^{-1}\left(\Lambda \overline{L_{k}}\right)^{T} \\
& +\sum_{k=1}^{r} e^{2 \delta\left(t-\tau-\mu_{m}\right)} \int_{t-\tau-\mu_{m}}^{t-\tau+\mu_{m}} \dot{z}^{T}(s) Z_{k} \dot{z}(s) d s
\end{aligned}
$$

Moreover, the time derivative of the functional $V\left(z_{t}\right)$ in
(20) is given by

$$
\begin{aligned}
\dot{V}\left(z_{t}\right)= & 2 \delta e^{2 \delta t} z^{T}(t) P z(t)+2 e^{2 \delta t} z^{T}(t) P \dot{z}(t) \\
& +2 e^{2 \delta t} \dot{z}^{T}(t) \int_{-\tau}^{0} Q z(t+\xi) d \xi \\
& +4 \delta e^{2 \delta t} z^{T}(t) \int_{-\tau}^{0} Q z(t+\xi) d \xi \\
& +2 e^{2 \delta t} z^{T}(t) Q z(t)-2 e^{2 \delta t} z^{T}(t) Q z(t-\tau) \\
& +\tau e^{2 \delta t} \bar{z}^{T}(t) \bar{R} \bar{z}(t)-\int_{t-\tau}^{t} e^{2 \delta s} \bar{z}^{T}(s) \bar{R} \bar{z}(s) d s \\
& +\left.e^{2 \delta(t+\xi)} z^{T}(t+\xi) S z(t+\xi)\right|_{-\tau} ^{0} \\
& +\sum_{k=1}^{r} 2 \mu_{m} e^{2 \delta t} \dot{z}^{T}(t) Z_{k} \dot{z}(t) \\
& -\sum_{k=1}^{r} \int_{t-\tau-\mu_{m}}^{t-\tau+\mu_{m}} e^{2 \delta s} \dot{z}^{T}(s) Z_{k} \dot{z}(s) d s
\end{aligned}
$$

Thus, assuming that $\bar{R}$ is given as in (16), $Z_{k}>0$ (implicit in (17)) and using (21), (23) and Jensen Inequality, the following upper bound for $\dot{V}\left(z_{t}\right)$ is obtained

$$
\begin{aligned}
\dot{V}\left(z_{t}\right) & \leq \zeta^{T} \Theta \zeta+\sum_{k=1}^{r} \mu_{m}\left(\Lambda \overline{L_{k}}\right) Z_{k}^{-1}\left(\Lambda \overline{L_{k}}\right)^{T} \\
& =\zeta^{T}\left(\Theta+\sum_{k=1}^{r} \Gamma_{k}\left(e^{2 \delta\left(\tau+\mu_{m}\right)} \mu_{m} Z_{k}\right)^{-1} \Gamma_{k}^{T}\right) \zeta
\end{aligned}
$$

with $\Theta$ given in (18), $\Gamma_{k}^{T}$ given in (19) and

$\zeta^{T}=\left[\begin{array}{llll}e^{\delta t} z^{T}(t) & e^{\delta t} \dot{z}^{T}(t) & e^{\delta t} z^{T}(t-\tau) & e^{\delta t} \int_{t-\tau}^{t} z^{T}(s) d s\end{array}\right]$.

Therefore, to guarantee that $\dot{V}\left(z_{t}\right)<0$ for any $\zeta \neq 0$ it suffices to impose that the term in the right side of (24) is negative. Moreover, using Schur's complement to impose that the term between parentheses in (24) is negative definite is equivalent to satisfy the LMI in (17). Then, if the LMI in (17) holds, the LKF time-derivative condition $\dot{V}\left(z_{t}\right)<$ $-\epsilon\|z(t)\|(\epsilon>0)$ is satisfied.

In what follows it is shown that if the LMIs in (15), (16) and (17) are satisfied, then the system in (4) achieves consensus with exponential convergence rate $\delta$. 
Using the inequality in (22) it follows that

$$
\begin{aligned}
2 e^{2 \delta t} z^{T}(t) & \int_{-\tau}^{0} Q z(t+\xi) d \xi \\
\leq & \int_{-\tau}^{0} z^{T}(t) e^{\delta t} Q I Q^{T} e^{\delta t} z(t) d \xi \\
& +\int_{t-\tau}^{t} e^{\delta t} z^{T}(s) I e^{\delta t} z(s) d s
\end{aligned}
$$

then the LKF has the following upper bound

$$
\begin{aligned}
V\left(z_{t}\right) \leq & e^{2 \delta t} \lambda_{\max }\{P\}\|z(t)\|^{2} \\
& +\tau e^{2 \delta t} \lambda_{\max }\left\{Q Q^{T}\right\}\|z(t)\|^{2}+e^{2 \delta t} \int_{t-\tau}^{t}\|z(s)\|^{2} d s \\
& +\tau \lambda_{\max }\{\bar{R}\} \int_{t-\tau}^{t} e^{2 \delta s}\|\bar{z}(s)\|^{2} d s \\
& +\lambda_{\max }\{S\} \int_{t-\tau}^{t} e^{2 \delta s}\|z(s)\|^{2} d s \\
& +\sum_{k=1}^{r}\left(\tau+\mu_{m}\right) \lambda_{\max }\left\{Z_{k}\right\} \int_{t-\tau-\mu_{m}}^{t} e^{2 \delta s}\|\dot{z}(s)\|^{2} d s \\
= & \bar{V}\left(z_{t}\right)
\end{aligned}
$$

where $\lambda_{\max }\{\cdot\}$ is the maximum eigenvalue of a matrix.

Assuming, that the LMIs in (15), (16) and (17) are satisfied, then $\dot{V}\left(z_{t}\right)<0$ and $V\left(z_{t}\right)>0$. Thus, it follows that

$$
0 \leq V\left(z_{t}\right) \leq V(z(0)) \leq\left.\bar{V}\left(z_{t}\right)\right|_{t=0} .
$$

Moreover, we have that

$$
\begin{aligned}
\bar{V}\left(z_{0}\right) \leq & \left\{\lambda_{\max }\{P\}+\tau \lambda_{\max }\left\{Q Q^{T}\right\}+\tau\right. \\
& \left.+\lambda_{\max }\{S\} \int_{-\tau}^{0} e^{2 \delta s} d s\right\} \sup _{-\tau \leq \theta \leq 0}\{\|z(\theta)\|\} \\
& +\left\{\tau \lambda_{\max }\{R\} \int_{-\tau}^{0} e^{2 \delta s} d s\right\} \sup _{-\tau \leq \theta \leq 0}\{\|\bar{z}(\theta)\|\} \\
+ & \left\{\left(\tau+\mu_{m}\right) \sum_{k=1}^{r} \lambda_{\max }\left\{Z_{k}\right\} \int_{-\tau-\mu_{m}}^{0} e^{2 \delta s} d s\right\} \\
& \times \sup _{-\left(\tau+\mu_{m}\right) \leq \theta \leq 0}\{\|\dot{z}(\theta)\|\}=\rho(\delta) .
\end{aligned}
$$

On the other hand,

$$
e^{2 \delta t} \lambda_{\min }\{P\}\|z(t)\|^{2} \leq V\left(z_{t}\right) \leq \rho(\delta),
$$

where $\lambda_{\min }\{P\}$ is the minimum eigenvalue of the matrix $P$. Thus, from (26), (27) and (28) it follows that

$$
\|z(t)\|^{2} \leq \frac{\rho(\delta)}{\lambda_{\min }\{P\}} e^{-2 \delta t} .
$$

Therefore, the multi-agent in (4) achieves consensus with exponential convergence rate $\delta$ according to Definition 1 . This completes the proof.

The exponential consensus analysis is important since it is often desired that the multi-agent system achieves consensus in an exponential rate to ensure fast response. In this context, the previous theorem is useful. However, the following result is appropriated when it is only necessary to verify whether the consensus is achieved or not. This result is obtained following the same steps presented in the proof of Theorem 1 and choosing an appropriate Lyapunov-Krasovskii functional as in (20) setting $\delta=0$.

Corollary 1: Let be given $\tau>0$ and $\mu_{m}$. Then the system in (4) with all $\tau_{k}(t) \in\left[\tau-\mu_{m}, \tau+\mu_{m}\right]$ achieves consensus, if there exist real matrices: $F, G, P=P^{T}, S=S^{T}, Q$, $R_{1}=R_{1}^{T}, R_{2}, R_{3}=R_{3}^{T}$ and $Z_{k}=Z_{k}^{T}, k=1,2, \ldots, r$, of dimension $(n-1) \times(n-1)$, such that the LMIs in (15), (16), (17) with $\delta=0$ are satisfied.

The next section presents a numerical example to show the effectiveness of the proposed methods.

\section{Numerical ExAmple}

Consider an undirected network of six agents with connections as presented in Fig.1.

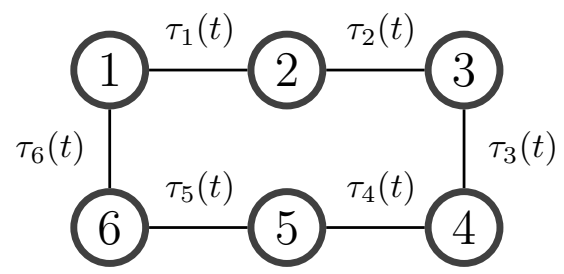

Fig. 1. Undirected interaction graph of the multi-agent system.

Initially, assuming that the time-delays are equal and constant, i.e. $\tau_{k}(t)=\tau$ for all $k$, the methods proposed in [5], [9], [7] and in Corollary 1 are used for comparison purpose. Then, the basic test is to find the largest time-delay $\tau$ such that the methods in [5], [9] and [7] and the proposed one can guarantee the consensus of the multi-agent system shown in Fig. 1. The results obtained are presented in Table I.

TABLE I

LARGEST CONSTANT TIME-DELAY $\tau$, ASSUMING $\tau_{k}(t)=\tau \forall k$.

\begin{tabular}{l|c}
\hline Method & constant time-delay $\tau$ \\
\hline [5, Th. 3] & 0.24 \\
[9, Th. 1] & 0.353 \\
[7, Th. 1] & 0.353 \\
Corollary 1 & 0.353 \\
\hline Analytical & 0.3925 \\
\hline
\end{tabular}

Notice that the results presented in Table I are neither favourable nor unfavourable to the proposed method. However, the methods in [9] and [7] can not be applied when the multi-agent system is subject to multiple non-differentiable time-varying delays in the interval $\tau_{k}(t) \in\left[\tau-\mu_{m}, \tau+\mu_{m}\right]$, where the $\tau-\mu_{m}>0$.

To illustrate the advantages of the proposed method, consider that the multi-agent system presented in Fig. 1 is subject to non-differentiable time-varying delays satisfying $\tau_{k}(t) \in\left[\tau-\mu_{m}, \tau+\mu_{m}\right]$, for $k=1,2, \ldots, 6$. Then, for given values of $\tau$ Corollary 1 is used to find the corresponding largest values of $\mu_{m}$. The results obtained are presented in Table II.

The results presented in tables I and II do not give any estimate of the time needed to achieve consensus. Therefore, 
TABLE II

LARGEST $\mu_{m}$ OBTAINED BY COROLLARY 1 FOR GIVEN $\tau$.

\begin{tabular}{c|c|c|c|c|c}
\hline$\tau$ & 0.15 & 0.20 & 0.25 & 0.30 & 0.35 \\
\hline$\mu_{m}$ & 0.095 & 0.070 & 0.047 & 0.024 & 0.001
\end{tabular}

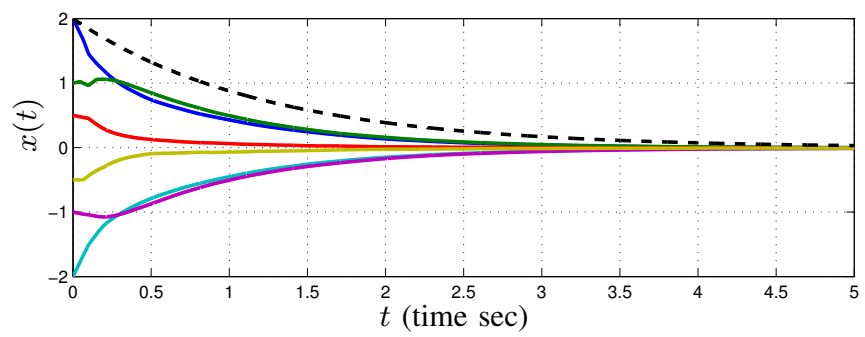

Fig. 2. The state trajectories of the agents for the multi-agent system in Fig. 1 subject to multiple time-varying delays such that all $\tau_{k}(t) \in$ $[\tau-\mu, \tau+\mu]$, for $k=1,2, \ldots, 6$, with $\tau=0.10$ and $\mu_{m}=0.06$ (solid lines) and an exponentially decreasing function $2 e^{-\delta t}$ with $\delta=0.82$ (dashed line).

the result in Theorem 1 is useful in this context. To illustrate that, consider the test of finding the largest value of the exponential decay rate $\delta$ for given pairs of $\tau$ and $\mu_{m}$, such that the multi-agent system achieves consensus with exponential decay rate $\delta$. The results obtained are shown in Table III.

TABLE III

LARGEST $\delta$ OBTAINED BY THEOREM 1 FOR GIVEN $\tau$ AND $\mu_{m}$.

\begin{tabular}{c|c|c|c}
\hline$\left(\tau, \mu_{m}\right)$ & $(0.10,0.06)$ & $(0.15,0.05)$ & $(0.20,0.04)$ \\
\hline$\delta$ & 0.82 & 0.79 & 0.58
\end{tabular}

Finally, for simulation sake we chose the delays as square wave function with random amplitude, such that all $\tau_{k}(t) \in$ $\left[\tau-\mu_{m}, \tau+\mu_{m}\right]$ for given $\tau$ and $\mu_{m}$. Then, the system time responses for two triplet $\left(\tau, \mu_{m}, \delta\right)$ in Table III are presented in figures 2 and 3. In these figures we can observe that the exponential convergence time gives a good estimate of the time needed for the system to achieve consensus. For illustration of the time-delays form used to obtain the time response presented, the Fig. 4 shows the delay $\tau_{1}(t)$ used.

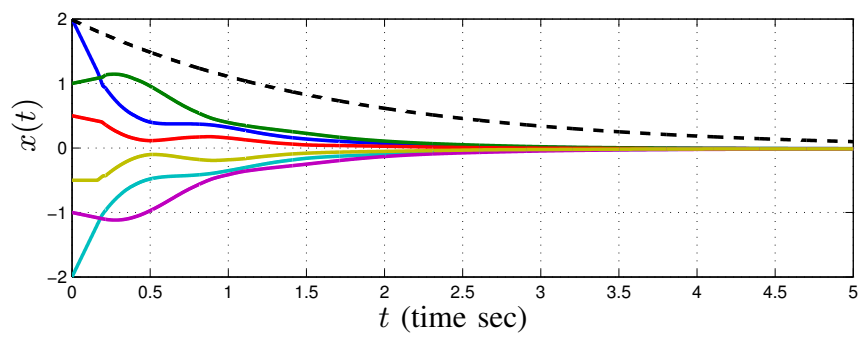

Fig. 3. The state trajectories of the agents for the multi-agent system in Fig. 1 subject to multiple time-varying delays such that all $\tau_{k}(t) \in$ $[\tau-\mu, \tau+\mu]$, for $k=1,2, \ldots, 6$, with $\tau=0.20$ and $\mu_{m}=0.04$ (solid lines) and an exponentially decreasing function $2 e^{-\delta t}$ with $\delta=0.58$ (dashed line).

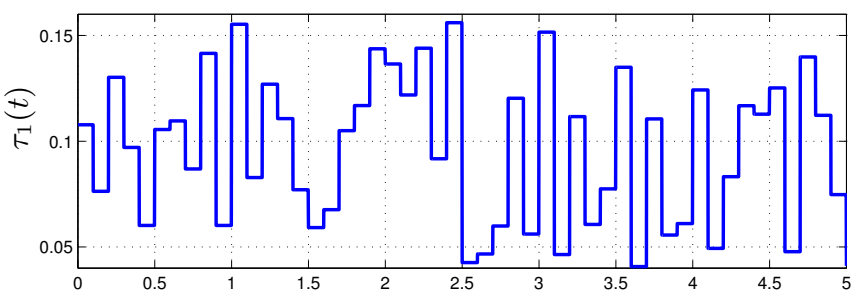

Fig. 4. Square wave function with random amplitude representing the time-varying delay $\tau_{1}(t)$.

\section{CONCLUSION}

LMI conditions which are sufficient to guarantee consensus for continuous-time multi-agent systems in the presence of time-delays has been derived. Multiple time-varying delays that can be non-differentiable and have minimum value greater than zero have been considered. This is a more general case since it allows to show consensus in different regions in the domain of time-delay.

Not only consensus problem has been dealt with but also a method to compute an estimate of the rate of convergence was proposed.

\section{REFERENCES}

[1] Y. Cao, W. Yu, W. Ren, and G. Chen, "An overview of recent progress in the study of distributed multi-agent coordination," IEEE Transactions on Industrial Informatics, special issue on Advances in Theories and Industrial Applications of Networked Control Systems, 2012, to appear.

[2] W. Ren, "On consensus algorithms for double-integrator dynamics," IEEE Transactions on Automatic Control, vol. 53, no. 6, pp. 1503$1509,2008$.

[3] Y. Li, J. Xiang, and W. Wei, "Consensus problems for linear timeinvariant multi-agent systems with saturation constraints," IET Control Theory and Applications, vol. 5, no. 6, pp. 823-829, 2011.

[4] R. Olfati-Saber and R. Murray, "Consensus problems in networks of agents with switching topology and time-delays," IEEE Transactions on Automatic Control, vol. 49, no. 9, pp. 1520-1533, 2004.

[5] P. Lin, Y. Jia, and L. Li, "Distributed robust $H_{\infty}$ consensus control in directed networks of agents with time-delay," Systems \& Control Letters, vol. 57, pp. 643-653, 2008.

[6] Y. Liu and Y. Jia, "Robust $H_{\infty}$ consensus control of uncertain multiagent systems with time delays," International Journal of Control, Automation and Systems, vol. 9, no. 6, pp. 1086-1094, 2011.

[7] C. Kecai, L. Chunxiang, G. Xiang, and H. Yang, "Consensus in multiagent with time-varying delays," in IEEE International Conference on Computer Science and Automation Engineering (CSAE), vol. 4, june 2011, pp. $313-317$.

[8] Q. Zhang, Y. Niu, L. Wang, L. Shen, and H. Zhu, "Average consensus seeking of high-order continuous-time multi-agent systems with multiple time-varying communication delays," International Journal of Control, Automation and Systems, vol. 9, no. 6, pp. 1209-1218, 2011.

[9] Y. G. Sun, L. Wang, and G. Xie, "Average consensus in networks of dynamic agents with switching topologies and multiple time-varying delays," Systems \& Control Letters, vol. 57, no. 2, pp. 175 - 183, 2008.

[10] Y. G. Sun and L. Wang, "Consensus of multi-agent systems in directed networks with nonuniform time-varying delays," IEEE Transactions on Automatic Control, vol. 54, no. 7, pp. 1607-1613, 2009.

[11] M. C. de Oliveira, F. O. Souza, and R. M. Palhares, "Assessing stability of time-delay systems using rational systems," in Proceeding of the 47th IEEE Conference on Decision and Control, Cancun, Mexico, 2008, pp. 4012-4017.

[12] C. Godsil and G. Royle, Algebraic Graph Theory. Springer-Verlag, 2001, vol. 207.

[13] K. Gu, V. Kharitonov, and J. Chen, Stability of time-delay systems. Boston, MA: Birkhuser, 2003. 